Artículo original

\title{
Factores que prolongan el proceso de referencia en salud entre una institución hospitalaria y un asegurador
}

\section{Factors prolonging the health referral process between a hospital institution and the regulatory center of an insurer}

\author{
Eliana Maritza Rojas Giraldo ${ }^{1 凶} \underline{\mathrm{ORCID}}$, Leidy Maryuri Zapata Ruiz $2 \llbracket \underline{\mathrm{ORCID}}$ \\ ${ }^{1}$ Médica y cirujana. Especialista en Gerencia de la Seguridad Social. Magister en Seguridad Social. Coordinadora médica de atención \\ salud nivel urgencias en EPS Sura regional Antioquia y eje cafetero. \\ ${ }^{2}$ Enfermera. Especialista en Cuidado a las personas con heridas y ostomías. Magister en Seguridad Social. Enfermera Clínica CES. \\ Coordinadora del servicio de clínica de heridas. Coordinadora académica especialización de Enfermería en cuidado de personas con \\ heridas y estomas, Universidad CES.
}

Fecha correspondencia:

Recibido: 23 de octubre de 2020.

Aceptado: 15 de marzo de 2021.

Forma de citar:

Rojas Giraldo EM, Zapata Ruiz

LM. Factores que prolongan el proceso de referencia en salud entre una institución hospitalaria y un asegurador. Rev CES Enf [Internet]; 2(2):40-59. Disponible en: https://dx.doi.org/10.21615/ cesenferm.5926

\section{Open access}

(C) Derecho de autor

Licencia creative commons

Ética de publicaciones

Revisión por pares

Gestión por Open Journal System DOI: $10.21615 /$ cesenferm.5926

ISSNe: 2745-049X

Publica con nosotros

\section{Resumen}

Objetivo: Establecer los factores demográficos y clínicos relacionados con el proceso de referencia en salud, entre una institución hospitalaria de alta complejidad y el centro regulador de un asegurador en una ciudad de Colombia. Metodología: Se analizaron 446 usuarios. Se compararon dos grupos según el tiempo de aceptación del proceso de referencia a la institución hospitalaria, articulándose al Decreto 4747 de 2007 y Resolución 3047 de 2008: menor a seis horas y mayor a seis horas en el proceso de referencia. Se realizó análisis estadístico aplicando medidas de frecuencias y prueba de Chi cuadrado con su correspondiente valor de p. Se calculó OR como medida de asociación para comparar ambos grupos con las variables independientes. Resultados: El 95,51\% de los datos analizados logró una respuesta a la remisión en menos de 6 horas, con una media de 1 hora y 59 minutos. Los usuarios dentro de este grupo se caracterizaron por ser menores a 26 y mayores a 60 años. Los pacientes con diagnósticos de enfermedades endocrinas, nutricionales y metabólicas, trastornos mentales y del comportamiento, enfermedades del aparato genitourinario y del 
sistema circulatorio están relacionados con remisiones más prolongadas; así, como los usuarios que tenían cuatro a seis comorbilidades. Igualmente, los factores como: iniciar la remisión desde una institución de alta complejidad, requerir una especialidad clínica, realizar el proceso en un día hábil, en horario nocturno y tener más de cuatro negaciones durante la solicitud favorecieron una duración mayor a seis horas para el proceso de referencia. Conclusiones: Algunas características demográficas, estado clínico del paciente y factores relacionados con el proceso de referencia, favorecieron remisiones con duración mayor a seis horas. Éste retraso puede relacionarse con la calidad y oportunidad de la atención en salud, y representa un reto tanto para las instituciones como para los centros reguladores. Se deben orientar actividades de mejora hacia resoluciones ágiles y efectivas que terminen favoreciendo el resultado en salud.

Palabras clave: traslado de pacientes; barreras de acceso; servicios de salud; aseguramiento en salud.

\section{Abstract}

Object: To establish the demographic and clinical factors related to the health referral process between a hospital institution and the regulatory center of an insurer in a Colombian city. Methods: We analyzed 446 users. Two groups were compared according to the acceptance time of the health referral process to the hospital institution, articulated with Decree 4747 of 2007 and Resolution 3047 of 2008: less than six hours and greater than six hours for the patient transfer. Statistical analysis was performed, applying the frequency and percentage measurements and the Chi-square test with its corresponding $p$-value. The epidemiological measure of prevalence ratios was calculated to compare both groups with the independent variables. Results: The analyzed data revealed that $95,51 \%$ of the users achieved a referral in less than 6 hours, with a mean of 1 hour and 59 minutes. This group's population was characterized as being under 26 and over 60 years of age. Patients with diagnoses of endocrine, nutritional and metabolic diseases, mental and behavioral disorders, diseases of the genitourinary system and circulatory system were associated with more extended referrals and users who had four to six comorbidities. Likewise, starting the referral from a highly complex institution, requiring a clinical specialty, carrying out the process in one business day, at night, and having more than four denials during the request, favored a duration greater than six hours for the referral process. Conclusions: Some demographic features, clinical states of the patients, and factors related to the referral process favored remissions lasting more than 6 hours. This delay can be related to the quality and timeliness of health care for users and 
represents a challenge for both institutions and regulatory centers. Improving activities should be aimed towards agile and effective resolutions that favor the health outcome.

Keywords: patient transfer; access barriers; health services; health insurance.

\section{Introducción}

El sistema de salud colombiano, establecido por la Ley 100 de 1993, creó el Sistema General de la Seguridad Social en Salud (SGSSS) en donde se instaura una afiliación obligatoria a través de las Entidades Administradoras de Planes de Beneficio (EAPB). Estas EAPB administran los recursos de las cotizaciones y garantizan los servicios del plan de beneficios en salud (PBS), teniendo acceso a estos planes por medio de las instituciones prestadoras de servicios de salud $\left(\right.$ IPS) ${ }^{(1)}$. Por ello, la relación entre las EAPB e IPS debe ser articulada permitiendo el logro de los objetivos particulares de cada una. Las EAPB son las responsables de garantizar la atención mediante la contratación y pago de servicios de salud públicos o privados, y por tanto de la configuración de las redes, con el primer nivel como puerta de entrada y un sistema de referencia para acceder a servicios adicionales ${ }^{(2)}$. Las IPS están organizadas en una red de atención y clasificadas de acuerdo con la complejidad de sus recursos ${ }^{(3)}$; son las encargadas de prestar servicios de salud con calidad, eficacia y eficiencia, permitiendo el logro de las necesidades de cuidado y restablecimiento de la salud de los individuos ${ }^{(4)}$.

La atención en salud de una persona y la identificación de la necesidad de un servicio adicional no disponible durante la atención es el punto de partida para la activación del proceso de referencia. Ya sea que se haga el requerimiento desde una cita programada, una cita prioritaria, la atención inicial de urgencias o desde servicios de internación, es el sistema de referencia el encargado de enviar un paciente o elementos de ayudas diagnósticas y/o terapéuticas por parte de un prestador de servicios de salud a otro para la atención o complementación diagnóstica de acuerdo con el nivel de complejidad, y en respuesta a las necesidades de salud solicitadas para dicho paciente ${ }^{(5)}$.

El proceso de referencia es el conjunto de procedimientos y actividades técnicos y administrativos que permiten coordinar adecuadamente los servicios de salud a los pacientes, con el objetivo de garantizar la calidad, accesibilidad, oportunidad, continuidad e integralidad de los servicios, en función de la organización de la red de prestación de servicios definida por la entidad responsable del pago ${ }^{(5)}$, siendo éste proceso donde confluyen e interactúan situaciones o características que influyen en el acceso oportuno a la autorización y atención en salud requerida ${ }^{(6)}$. En Colombia, el proceso de referencia está incluido en las leyes de salud 
como lo son la Constitución Política colombiana, la Ley 100 de 1993, el Decreto 4747de 2007, la Resolución 3047 de 2008 y la Resolución 4331 de $2012^{(7)}$.

Desde la legislación, se contempla que el asegurador o entidad responsable del pago, cuenta con un tiempo de seis horas para dar respuesta o autorización a las IPS sobre la solicitud de servicios adicionales o remisión para atención complementaria posterior a la atención inicial de urgencias o desde servicios de internación ${ }^{(8)}$. En el marco actual de relacionamiento, el cual se basa en convenios entre aseguradores e IPS, este tiempo marca un límite importante para que el asegurador en salud pueda transferir el usuario a su red contratada, ya sea para continuar con la atención o para cumplir con las solicitudes desde la IPS remitente por no disponibilidad o por corresponder a un menor nivel de atención.

Aunque existe literatura para describir localmente los procesos de referencia ${ }^{(9,10)}$, no hay una pauta clara desde la legislación para calificar como oportuna o no la duración de dicho proceso. Se ha limitado dicho tiempo a ciertos diagnósticos, que sin intervenciones tempranas ${ }^{(11)}$ generarían secuelas graves o alta probabilidad de mortalidad. Estos tiempos están basados en guías clínicas de asociaciones médicas internacionales. Sin embargo, no se cuenta con evidencia a nivel local, que tenga en cuenta las características específicas del contexto. Adicionalmente, no se cuenta con estudios que midan el tiempo de respuesta y que lo califique como oportuno o no ${ }^{(7,12)}$.

Un sistema de referencia articula los tres niveles de atención para ofrecer al usuario recursos y tecnología necesarias para resolver su situación de salud ${ }^{(13)}$, por ello es importante identificar características en la población y en el proceso para entender cuáles favorecen que la remisión sea prolongada o no, y cuáles pueden intervenirse para mitigar las dificultades y mejorar los tiempos de respuesta entre las redes de atención. Estudios han reportado algunas características que influyen en el proceso de referencia; la edad mayor de 65 años, ser mujer, tener entre las principales causas de remisión una patología respiratoria o gastrointestinal, hacer solicitudes los viernes y en el horario de 14 a 21 es más frecuente el proceso de remisión $\mathrm{y}$ menos frecuentes los domingos $\mathrm{y}$, con respecto al tiempo, no tener convenio con las instituciones, favorecen una remisión prolongada ${ }^{(14-16)}$.

Esta investigación tuvo como objetivo establecer los factores demográficos y clínicos, relacionados con el proceso de referencia en salud, entre una institución hospitalaria y el centro regulador de un asegurador, en una ciudad de Colombia. 


\section{Materiales y métodos}

Se realizó un estudio con diseño transversal observacional, de intensión analítica con fuente de datos secundaria. La población estudiada corresponde a registros de pacientes de una EAPB en proceso de remisión, aceptados y atendidos en una IPS de alto nivel de complejidad en el segundo semestre de 2018. La selección de la IPS se realizó considerando que ésta concentraba el mayor porcentaje de hospitalizados del asegurador en el área metropolitana del Valle de Aburrá en la ciudad de Medellín.

La información se recolectó de las bases de datos elaboradas por la entidad aseguradora y la entidad hospitalaria receptora, respectivamente, durante cada uno de los procesos de referencia. La información recopilada por el asegurador contenía 4882 registros, los cuales fueron comparados con aquellos entregados por la institución hospitalaria, la cual tuvo 7112 registros para referencia. Se seleccionaron los registros donde coincidieron los datos del respectivo proceso en ambas entidades. Se revisó cada dato buscando inconsistencias, inexistencias o información no comprobable para excluir el dato del estudio. Cada dato se comparó en ambas bases de datos, tomando los que tenían los mismos valores y eliminando aquellos que no, para no afectar la validez del estudio.

Posterior a la depuración, se obtuvo una muestra con 446 registros de pacientes, los cuales contenían toda la información necesaria para caracterizar los usuarios y su necesidad particular en salud, y definir las variables. En el programa Microsoft ${ }^{\circledR}$ Excel $^{\circledR} 2016$ para Windows (Licencia Universidad (ES), se consolidó una nueva base de datos, donde se conjugaron los datos extraídos.

Las variables que se obtuvieron de la base de datos del asegurador fueron edad, sexo, régimen del afiliado, número de comorbilidades, triage y complejidad de la entidad remitente, especialidad solicitada, número de negaciones, día, horario y grupos diagnósticos. Adicionalmente, se contó con el dato del número de procesos de referencia de cada paciente previos al evento estudiado, considerando este dato como relevante en relación con la utilización por parte del usuario del sistema y sus necesidades en salud. Las variables derivadas de la base de datos de la IPS fueron destino en la institución y el tiempo entre la solicitud y el ingreso a la IPS hospitalaria receptora.

Se construyó además, una variable dependiente cualitativa dicotómica que se nombró como: tiempo para aceptación menor a seis horas y mayor a seis horas. El corte de tiempo en seis horas se estableció teniendo en cuenta el Decreto 4747 de 2007 y la Resolución 3047 de 
$2008^{(5,8)}$ donde se hace mención, que para la realización de servicios posteriores a la atención de urgencias y en caso de requerir servicios adicionales (referencia), la aseguradora tendrá que dar respuesta a la IPS que atiende, dentro de las 6 horas siguientes al recibo de la solicitud, considerando así, este tiempo como el adecuado para dar respuesta a las necesidades en salud y atención del usuario. El tiempo mayor a 6 horas se consideró como un proceso de referencia prolongado.

El acceso a ambos archivos tuvo su respectiva autorización de uso y aval del comité de ética de cada entidad, gestionados en noviembre de 2019. El proceso se ajustó a las normas éticas de investigación en seres humanos, consignadas en la Resolución 8430 de $1993^{(17)}$, teniendo en cuenta que es un estudio con fuente secundaria sin riesgo. También se pacta el compromiso de confidencialidad, protocolo de custodia de datos y compromiso de reserva de nombres y razones sociales de las entidades participantes. A partir de esta recopilación, se caracterizó la población en términos demográficos, perfil de morbilidad y aseguramiento.

Se realizó análisis estadístico de los datos, aplicando las medidas de frecuencia absolutas y frecuencias relativas; se realizó prueba de Chi cuadrado y su correspondiente valor de $p$ con un nivel de significancia de 0,05. Finalmente se calculan OR (Odss Ratio) para comparar ambos grupos con las variables independientes ${ }^{(18)}$. La organización y el análisis de los datos se realizó con ayuda del programa Microsoft ${ }^{\circledR}$ Excel $^{\circledR} 2016$ para Windows y Epidat versión 4.2 y 3.1 de distribución gratuita.

\section{Resultados}

Se analizaron 446 registros de pacientes pertenecientes a un mismo asegurador en salud, en quienes posterior a la atención inicial de urgencias o durante su estancia en hospitalización, en diferentes instituciones, se inició un proceso de referencia y fueron efectivamente aceptados por la IPS hospitalaria que participó en este estudio. El grupo de edad más frecuente correspondió a las personas entre 60 a 107 años de edad con un 51,8\% de participación.

La distribución por sexo fue similar, aunque ligeramente mayor para el sexo masculino (50,7\%). El $88,6 \%$ de la población remitida tenía entre cero a tres antecedentes patológicos concomitantemente, el 58,1\%, previo al evento estudiado, ya registraba entre uno a cinco procesos de remisión, y el 8,5\% tenía más de 21 procesos de remisión en su histórico de afiliación al asegurador. Los diagnósticos con mayor número de remisiones fueron las enfermedades e infecciones del sistema respiratorio en un $20,2 \%$, seguido de enfermedades del aparato digestivo 18,6\%. Dentro de los otros diagnósticos con menor presencia en la 
Julio - diciembre de 2021

muestra fueron los siguientes con su respectivo porcentaje; enfermedades del sistema osteomuscular y del tejido conectivo en un $1,12 \%$, enfermedades del ojo y sus anexos al igual que enfermedades del sistema nervioso en un $0,67 \%$ y finalmente neoplasias en un $0,22 \%$.

El destino más frecuente en la IPS a la cual se remitieron los usuarios, fue al servicio de hospitalización con un porcentaje del $90,6 \%$ y el resto a una unidad de alta dependencia. Según las características de aseguramiento, se encontró que la mayor proporción de personas eran del régimen contributivo $(96,6 \%)$ el resto pertenecían al régimen subsidiado (3,4\%). De la población del régimen contributivo, la mayoría contaba con más de 520 semanas cotizadas $(47,5 \%)$ seguida de la población que tenía entre 105 a 260 semanas cotizadas con un $22 \%$ (Tabla 1).

Tabla 1. Descripción de la Población con proceso de referencia entre un asegurador y un prestador de servicios de salud.

\begin{tabular}{|c|c|c|}
\hline Características & $\begin{array}{c}\text { Frecuencia } \\
\text { Absoluta }\end{array}$ & Porcentaje \\
\hline \multicolumn{3}{|l|}{ Edad } \\
\hline Menor a 26 años & 45 & $10.1 \%$ \\
\hline 27 a 59 años & 170 & $38.1 \%$ \\
\hline 60 a 107 años & 231 & $51.8 \%$ \\
\hline \multicolumn{3}{|l|}{ Sexo } \\
\hline Femenino & 220 & $49.3 \%$ \\
\hline Masculino & 226 & $50.7 \%$ \\
\hline \multicolumn{3}{|l|}{ Ubicación de IPS de afiliación } \\
\hline Medellín y área metropolitana & 441 & $99 \%$ \\
\hline Otros municipios & 5 & $1 \%$ \\
\hline \multicolumn{3}{|l|}{ Comorbilidades } \\
\hline 0 a 3 & 395 & $88.6 \%$ \\
\hline 4 a 6 & 51 & $11.4 \%$ \\
\hline \multicolumn{3}{|l|}{ Número de remisiones del usuario } \\
\hline 1 a 5 & 259 & $58.1 \%$ \\
\hline 6 a 10 & 86 & $19.3 \%$ \\
\hline 11 a 20 & 63 & $14.1 \%$ \\
\hline Mayor a 21 & 38 & $8.5 \%$ \\
\hline \multicolumn{3}{|l|}{ Destino en la IPS que acepta } \\
\hline Hospitalización & 404 & $90.58 \%$ \\
\hline UCE/UCI & 42 & $9.42 \%$ \\
\hline \multicolumn{3}{|l|}{ Diagnósticos de remisión } \\
\hline Enfermedades del sistema respiratorio & 90 & $20.2 \%$ \\
\hline Enfermedades del aparato digestivo & 83 & $18.6 \%$ \\
\hline
\end{tabular}




\begin{tabular}{lcc}
\hline \multicolumn{1}{c}{ Características } & $\begin{array}{c}\text { Frecuencia } \\
\text { Absoluta }\end{array}$ & Porcentaje \\
\hline Sin Clasificación por sistemas & 79 & $17.7 \%$ \\
\hline Ciertas enfermedades infecciosas y parasitarias & 45 & $10.1 \%$ \\
\hline Enfermedades del sistema circulatorio & 33 & $7.4 \%$ \\
\hline Trastornos mentales y del comportamiento & 32 & $7.2 \%$ \\
\hline Enfermedades del aparato genitourinario & 27 & $6.1 \%$ \\
\hline Enfermedades de la piel y el tejido subcutáneo & 21 & $4.7 \%$ \\
\hline Traumatismos, envenenamientos y algunas otras & 15 & $3.4 \%$ \\
consecuencias de causa externa & 12 & $2.7 \%$ \\
\hline Otros grupos diagnósticos & 9 & $2.0 \%$ \\
\hline Enfermedades endocrinas, nutricionales y & & \\
metabólicas & & $96.6 \%$ \\
\hline Régimen de afiliación & 431 & $3.4 \%$ \\
\hline Contributivo & 15 & \\
\hline Subsidiado & & $9.6 \%$ \\
\hline Semanas cotizadas & 43 & $7.8 \%$ \\
\hline 0 a 52 & 35 & $22 \%$ \\
\hline 53 a 104 & 98 & $13 \%$ \\
\hline 105 a 260 & 58 & $47.5 \%$ \\
\hline 261 a 520 & 212 & \\
\hline$>520$ & &
\end{tabular}

Respecto al tiempo de aceptación, los procesos mayores a 6 horas representaron el 4,48\% de la población con un tiempo para remisión promedio de 16 horas. El porcentaje restante, 95,51\% tuvo un proceso de referencia menor a las 6 horas, con una media de 1 hora y 18 minutos. Toda la muestra obtuvo una media de 1 hora y 59 minutos entre la solicitud y la respuesta de la remisión. En cuanto al tiempo entre la respuesta a la remisión por la EAPB y la llegada a la IPS hospitalaria, toda la población tuvo una media de 4 horas y 45 min con un mínimo de una hora y máximo de 13 horas (Tabla 2).

Se encontró que algunos factores demográficos de la población influyeron en el proceso de referencia. Teniendo en cuenta la edad, quienes se encontraban en una edad menor de 26 años y entre 60 a 107 años, fueron los grupos con menor probabilidad de presentar tiempo prolongado; por el contrario, aquellos con edades entre 27 y 59 años tuvieron un $66 \%$ de mayor probabilidad de tener procesos más demorados $(O R=1,66)$ con respecto a los menores de 26 años. En relación con el sexo, los hombres tuvieron $20 \%$ mayor probabilidad de retraso en el proceso de referencia con respecto a las mujeres $(O R=1,20)$ ( $\underline{\text { Tabla } 3}$ ). 
Tabla 2. Tiempo del proceso de remisión e ingreso a la IPS hospitalaria receptora.

\begin{tabular}{|c|c|c|c|c|c|c|}
\hline & \multicolumn{2}{|c|}{ Total Muestra } & \multicolumn{2}{|c|}{ Menor a $6 h$} & \multicolumn{2}{|c|}{ Mayora $6 h$} \\
\hline & $\begin{array}{l}\text { Tiempo para } \\
\text { aceptación } \\
\text { (hh:mm:ss) }\end{array}$ & $\begin{array}{c}\text { Tiempo } \\
\text { desde } \\
\text { aceptación } \\
\text { hasta } \\
\text { ingreso a la } \\
\text { IPS }\end{array}$ & $\begin{array}{l}\text { Tiempo para } \\
\text { aceptación } \\
\text { (hh:mm:ss) }\end{array}$ & $\begin{array}{l}\text { Tiempo desde } \\
\text { aceptación } \\
\text { hasta ingreso } \\
\text { a la IPS }\end{array}$ & $\begin{array}{l}\text { Tiempo para } \\
\text { aceptación } \\
\text { (hh:mm:ss) }\end{array}$ & $\begin{array}{l}\text { Tiempo } \\
\text { desde } \\
\text { aceptación } \\
\text { hasta } \\
\text { ingreso a la } \\
\text { IPS }\end{array}$ \\
\hline Media & 01:59:43 & $04: 45: 37$ & 01:18:38 & 04:39:46 & $16: 34: 44$ & $06: 50: 15$ \\
\hline Mediana & 01:00:31 & 04:08:44 & $00: 58: 23$ & 03:58:58 & $11: 48: 43$ & 05:46:51 \\
\hline Mínima & 00:00:58 & 01:00:49 & 00:00:58 & 01:00:49 & $06: 30: 38$ & 01:37:35 \\
\hline Cuartil 25 & 00:33:01 & $02: 29: 13$ & $00: 32: 13$ & $02: 27: 54$ & 08:01:08 & 04:21:03 \\
\hline Cuartil 50 & 01:00:31 & 04:08:44 & $00: 58: 23$ & 03:58:58 & $11: 48: 43$ & $05: 46: 51$ \\
\hline Cuartil 75 & $01: 54: 27$ & $06: 20: 54$ & 01:43:50 & 06:19:01 & $24: 20: 31$ & 09:35:00 \\
\hline Máxima & $61: 22: 27$ & $12: 59: 35$ & $05: 56: 33$ & $12: 59: 35$ & $61: 22: 27$ & $12: 35: 33$ \\
\hline
\end{tabular}

En cuanto al estado de salud de la población, teniendo en cuenta el número de comorbilidades, se encontró que las personas que presentaban de 4 a 6 comorbilidades, tuvieron casi tres veces la probabilidad de tener un proceso de referencia mayor a 6 horas, comparado con los que tenían tres o menos comorbilidades $(\mathrm{OR}=2,75)$.

Según los diagnósticos de remisión que presentaron un proceso de referencia mayor a 6 horas, se encontró que aquellas personas con enfermedades endocrinas, nutricionales y metabólicas tuvieron casi siete veces $(O R=6,65)$ la probabilidad de presentar retrasos; los pacientes con trastornos mentales y del comportamiento tuvieron tres veces la probabilidad $(\mathrm{OR}=3,55)$; al igual que los pacientes con enfermedades del aparato genitourinario $(O R=2,95)$ y del sistema circulatorio $(O R=2,33)$. Diagnósticos relacionados con Enfermedades de la piel y el tejido subcutáneo $(O R=1,07)$ tuvieron una probabilidad de $7 \%$ en retraso para el proceso de referencia.

En adición y según la clasificación del triage realizado por la entidad remitente quienes fueron clasificados triage uno, tuvieron una probabilidad de demora del $65 \%(\mathrm{OR}=1,65)$, comparado con los pacientes clasificados en triage dos ( $\underline{\text { Tabla } 3}$ ). 
Tabla 3. Tiempo del proceso de referencia entre un asegurador y un prestador de servicios de salud.

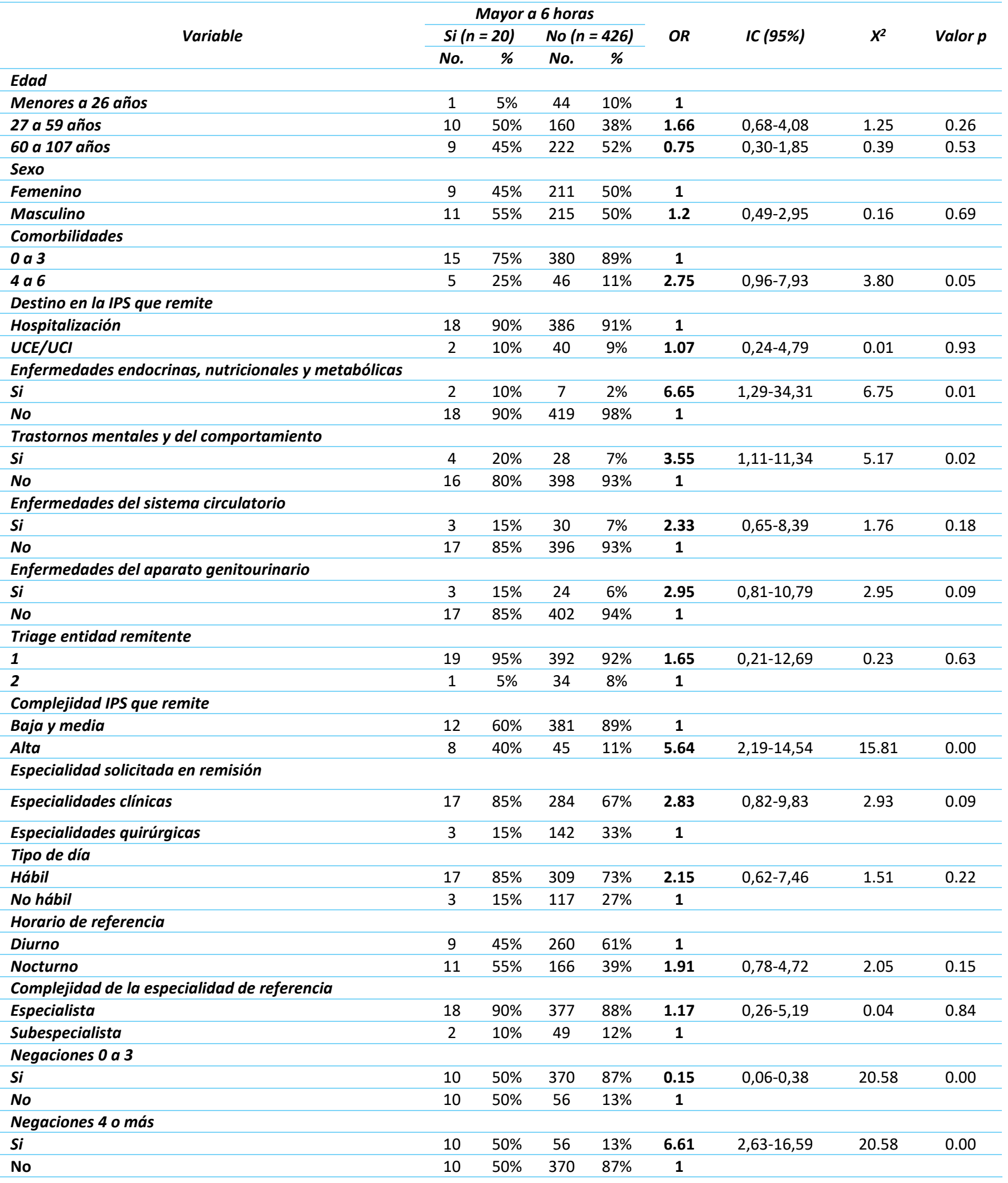


Teniendo en cuenta los factores propios relacionados con la atención y el proceso de referencia se observó que las remisiones realizadas en días hábiles tuvieron dos veces la probabilidad de demora en el proceso de referencia con respecto a las que se presentaron en los días no hábiles $(O R=2,15)$. Los pacientes que fueron remitidos en horarios nocturnos, tuvieron un $91 \%$ mayor probabilidad de durar más de seis horas en este proceso, comparado con los pacientes atendidos en horario diurno $(O R=1,91)$. Seguidamente, según la especialidad solicitada en remisión, las especialidades clínicas tuvieron tendencia a tener tiempos más prolongados con casi tres veces más probabilidad de demora en comparación con las especialidades quirúrgicas (Tabla 3).

Las remisiones que se iniciaron en IPS de alta complejidad, tuvieron casi seis veces más probabilidad de tener procesos prolongados presentando una diferencia significativa $(p<0,00)$ en comparación con aquellas IPS de baja y media complejidad. En cuanto al servicio de internación en la IPS que aceptó el paciente, quienes ingresaron a unidades de alta dependencia (UCE/UCI), tuvieron una probabilidad del $7 \%$ de retraso en el proceso de referencia en comparación con aquellos que ingresaron al servicio de hospitalización en sala general.

Finalmente, según las negaciones documentadas durante el proceso de referencia, se encontró que quienes tenían más de cuatro negaciones, tuvieron casi siete veces la probabilidad de presentar tiempos prolongados mayores a seis horas, con una diferencia significativa $(p<0,00)$ con respecto a quienes tenían menos de cuatro negaciones $(O R=6,61)$ (Tabla 3). Estas negaciones fueron previas a la aceptación en la IPS hospitalaria participante en el estudio, dentro de las cuales se identificó la no disponibilidad de camas como la principal causa.

\section{Discusión}

El actual Sistema General de Seguridad Social en Salud contempla en sus procesos la descentralización por niveles de atención y la comunicación entre ellos a través del sistema de referencia y contrareferencia para la atención de las necesidades en salud de la población a través de convenios con las EAPB, quienes deberán contar con un plan básico de salud y canales que permitan el acceso a los servicios de alta complejidad a sus afiliados en articulación con la red de IPS garantizando la calidad, accesibilidad, oportunidad, continuidad e integralidad en la atención ${ }^{(19)}$. Según los hallazgos de este estudio, se han encontrado situaciones o características que permiten llevar a cabo un proceso de referencia prolongado o no, en el contexto de la relación entre una EAPB y su principal IPS hospitalaria de referencia de alta complejidad que pueden ser útiles para identificar oportunidades de mejora para el sistema de salud y sus actores. 
El proceso de referencia fue más frecuente en hombres que en mujeres con un $0,7 \%$ de diferencia entre ellos, coincidiendo con otros estudios locales ${ }^{(20)}$. Sin embargo, esta condición cambia al incluir población geriátrica donde asciende en mujeres y disminuye en hombres ${ }^{(14,16)}$. Hay estudios que muestran una diferencia más significativa, de hasta el $22 \%{ }^{(3)}$, dependiendo de los rangos de edad incluidos.

La edad con mayor frecuencia encontrada en los procesos de referencia revisados fueron los mayores de 60 años, siendo 107 años la edad máxima encontrada. Hallazgo que concuerda con estudios del Reino Unido, donde reportan que el mayor porcentaje de remisiones por grupo de edad, fueron los adultos mayores de 80 años, con cifras de hasta el 41,3\% respecto a otras edades, evidenciándose que por cada diez años más de edad se aumentaba un 19\% la probabilidad de requerir traslado a un mayor nivel de atención ${ }^{(20)}$. Al comparar con otros estudios que excluía población pediátrica, la frecuencia se concentra en la población menor a la edad normativa para pensión en Colombia, coincidiendo en tener mayor frecuencia en edad productiva, entre 19 y 65 años ${ }^{(21)}$.

Los diagnósticos más frecuentes en este estudio que motivaron iniciar el proceso de referencia fueron las enfermedades e infecciones del sistema respiratorio seguido de enfermedades del aparato digestivo, resultado que coincide con otros estudios donde se encontró como primeras causas entre otras patologías, la agudización de enfermedades respiratorias y alteraciones digestivas y/o del ritmo intestinal ${ }^{(14)}$. Otro estudio encontró que las patologías no quirúrgicas fueron el principal motivo para el proceso de referencia $(69 \%)^{(3)}$ y otro estudio realizado en Chile evidencia, también la patología respiratoria como uno de los principales diagnósticos de remisión a pesar de realizarse en población pediátrica ${ }^{(22)}$.

Por otra parte, los diagnósticos que presentaron mayor probabilidad de presentar procesos de referencia mayor a seis horas, fueron las enfermedades endocrinas, nutricionales $y$ metabólicas, los pacientes con trastornos mentales y del comportamiento, enfermedades del aparato genitourinario y del sistema circulatorio, lo cual puede deberse a lo encontrado en un estudio que reporta en las IPS de mediana y alta complejidad, la no disponibilidad de médicos especialistas $(55,6 \%)$ y el cierre de servicios especializados $(41,3 \%)$, lo que afecta la oferta de sus servicios en salud. Además, mencionan entre otras causas de la disminución de médicos especialistas la poca formación de médicos en áreas especializadas (73\%) e insuficiencia de cupos para especializarse $(60 \%)^{(23)}$. 
En Colombia, el 23,1\% de los médicos graduados se especializa, para el 2016 la oferta de médicos especialistas en áreas clínicas, quirúrgicas y diagnósticas se estimó en 23,315 y en su mayoría correspondió a medicina interna (14\%), para el 2030 la oferta será de 45,789. Para el 2016 la demanda de médicos especialistas fue de 39 mil, siendo el 60\% de ella, especialidades clínicas, y medicina interna la de mayor demanda (13\% de los especialistas). Para el 2030 la demanda será de 52,568 ${ }^{(24)}$. Teniendo en cuenta lo anterior y que los especialistas en medicina interna pueden optar por la formación en subespecialidad como endocrinología, cardiología, entre otros, se reduce aún más la oferta con relación a esta especialidad y sus subespecialidades. Además, un estudio en Boston orientado a identificar los más afectados en los procesos de remisión, encontró un promedio de dos días para el proceso de referencia a un centro de hospitalización general con disponibilidad de algunas subespecialidades. La incertidumbre de la duración de tal proceso fue una inconformidad manifestada tanto por el paciente como por los médicos tratantes, asociado a la inoportunidad de valoraciones especializadas, especialmente en aquellos usuarios con traslado nocturno a las instituciones ${ }^{(25)}$.

En cuanto a las comorbilidades de las personas de este estudio, quienes tenían concomitantemente de cuatro a seis comorbilidades tuvieron mayor probabilidad de tener procesos de remisión más prolongados; lo anterior, hace pensar en que las instituciones no solo fijan su atención en el motivo principal que conlleva al proceso de referencia, sino, que hacen un análisis integral de esta persona teniendo en cuenta sus comorbilidades y proyectando la necesidad de especialistas adicionales para su atención con los cuales en determinado momento pueden no disponer. Seguidamente, las personas que presentan varias enfermedades crónicas relevantes al mismo tiempo, en el ámbito medico son llamados pacientes pluripatológicos $y$, en ocasiones, una de sus comorbilidades o varias de ellas condicionan una dependencia importante en las actividades básicas de la vida diaria, y con lleva, un grado de complejidad, polifarmacia, hiperfrecuentación y deterioro funcional progresivo característicos. Siendo importante a la hora de requerir una admisión en un servicio hospitalario contar con una valoración multidimensional y multidisciplinaria donde no solo sea importante el motivo de remisión específico sino todas sus patologías concomitantes, para lo cual se debe tener instituciones de salud que cuenten con todo el recurso humano para su atención ${ }^{(26)}$.

Iniciar un proceso de referencia un día hábil en horario nocturno tiene una alta probabilidad de demora para más de seis horas de aceptación por otra institución. Un panorama no alejado de los hallazgos en estudios similares. La literatura disponible ya ha descrito el "efecto fin de semana" (weekend effect), el cual se relaciona con peores resultados en salud (27). Aunque los 
datos expuestos en este trabajo nos orientan a que hay menor demora en el proceso de referencia los días no hábiles, no es ajeno que el fin de semana disminuyen las aceptaciones en las instituciones ${ }^{(28)}$, debido a la disminución de recurso humano que es exclusivo de los días hábiles. No sucede igual con las solicitudes de referencia durante el fin de semana, las cuales pueden disminuir en número, pero la criticidad del enfermo puede ser mayor. Un estudio realizado en Boston E.U con 24,352 pacientes, encontró que las referencias realizadas en fines de semana, especialmente domingo en la noche, tuvieron mayor probabilidad de complicaciones clínicas y muerte respecto a las realizadas los días lunes en horario diurno ${ }^{(29)}$. Definitivamente, independiente de la frecuencia encontrada en los estudios, se considera que las remisiones realizadas durante fin de semana, sin tener en cuenta el tiempo de aceptación, es un predictor independiente de mortalidad (30).

Un hallazgo importante en el presente estudio se relaciona con que, la referencia originada desde un nivel bajo y medio de complejidad toma menos tiempo de aceptación que una originada desde la alta complejidad. Se ha concebido que el nivel de atención de baja complejidad tiene una resolutividad de hasta el $43 \%$ de su demanda de atención; también se ha descrito que éste nivel tiene procesos de atención y referencia más ágiles, traduciéndose en menores tiempos de estancia ${ }^{(31)}$. Además, las necesidades en salud que motivan las remisiones desde la baja complejidad, son más puntuales y concretas lo que les permite a las instituciones que revisan los casos, tomar decisiones en menor tiempo y aceptar los pacientes ${ }^{(32)}$. Otros estudios contemplan una frecuencia hasta de un 25,9\% de necesidad de referencia desde unidades de atención de urgencias básicas a niveles superiores de atención. Reforzando el concepto de alta resolutividad del nivel bajo de complejidad ${ }^{(20)}$. La amplia oferta de servicios disponibles en alta complejidad es inversamente proporcional a la frecuencia de remisiones originadas allí. Se ha descrito que desde estas IPS hay una frecuencia de remisiones del $2,9 \%{ }^{(3)}$.

En la literatura se han reportado algunas barreras para el acceso a los servicios de salud a nivel inferior o superior como la creciente demanda de recursos en salud, la oferta limitada de la red hospitalaria, el colapso de los servicios de urgencias, (33) la insuficiente disponibilidad del recurso humano especialmente el subespecializado, la restringida infraestructura en el momento requerido dada por una situación específica en salud, trámites administrativos internos y externos, la escasa disponibilidad de servicios de traslados, remisiones innecesarias por no pertinencia asociada a enfoques clínicos inadecuados, información insuficiente del usuario y asimetría de la información ${ }^{(2)}$. Situaciones que podrían convertirse en una causa de negación durante el proceso de remisión que prolongarán el tiempo en el cual la persona debe ser ubicada en una institución de salud de acuerdo a sus necesidades de atención. 
Estudios adicionales identificaron otros factores que pueden contribuir para un funcionamiento adecuado del proceso de referencia como: existencia de guías para el médico que definan los criterios y lugares de derivación de acuerdo con la capacidad resolutiva de las instituciones, realización de paraclínicos especializados que sirvan para definir la posterior valoración del especialista y para disminuir el tiempo de atención, la calidad de los registros médicos que permitan interpretar la situación del paciente ${ }^{(34)}$, y un sistema de retroalimentación que facilita la comunicación y el intercambio de información entre profesionales (referencia/contrareferencia, informe de alta hospitalaria, historia clínica compartida). Su existencia y uso adecuado permiten lograr la integralidad y mejorar la calidad de la atención en salud ${ }^{(35)}$.

\section{Limitaciones del estudio}

Inicialmente, el presente estudio se planteó investigar el proceso de referencia y contrareferencia. Sin embargo, en el momento de solicitar las bases de datos, se identificó que tanto la IPS hospitalaria como el centro regulador del asegurador, asumían la contrareferencia como un nuevo proceso de remisión para complementar la atención del paciente en el mismo evento de salud. La calidad en el diligenciamiento de las fichas de remisión de los usuarios por la IPS hospitalaria y el centro regulador del asegurador, fue un aspecto que limitó el tamaño de la muestra. Los estudios relacionados con el tiempo de duración del proceso de referencia fueron escasos y no permitió una discusión más amplia.

\section{Conclusiones}

En las características demográficas revisadas, la población menor a 26 años y mayor a 60 años, se relacionó con procesos de referencia de seis horas o menos. Los factores clínicos de los pacientes remitidos, específicamente algunos diagnósticos como enfermedades endocrinas, nutricionales y metabólicas, los trastornos mentales y del comportamiento, las enfermedades del aparato genitourinario y del sistema circulatorio están relacionados con un proceso de remisión mayor a seis horas. Al igual que las personas que tenían cuatro a seis comorbilidades concomitantemente.

Durante el análisis de los factores relacionados con el proceso de referencia, se identificó que algunos favorecieron una duración mayor a seis horas, tales como: iniciar la remisión desde una IPS de alta complejidad, requerir una especialidad clínica, realizar el proceso en un día hábil, en horario nocturno y tener más de cuatro negaciones durante la solicitud. El tiempo de duración de un proceso de referencia puede relacionarse con la calidad y oportunidad de la atención en salud a los usuarios, y representa un reto tanto para las instituciones como para los centros 
reguladores, para orientar sus actividades a resoluciones ágiles y efectivas que terminen favoreciendo el resultado en salud.

\section{Conflicto de interés}

Los autores declaran no tener ningún conflicto de interés.

\section{Referencias}

1. Bernal O, Barbosa S. La nueva reforma a la salud en Colombia: el derecho, el aseguramiento y el sistema de salud. Salud Pública Méx. 2015;57:433-40.

2. León-Arce HG, Mogollón-Pérez A-S, Vargas I, Vázquez M-L. Factores que influyen en el uso de mecanismos de coordinación entre niveles asistenciales en Colombia. Gac Sanit [Internet].2019; Disponible

en: http://www.sciencedirect.com/science/article/pii/S0213911119301724

3. Giraldo J, Peña T, Naranjo A, Lopera Y. Caracterización de los traslados médicos no regulados desde diferentes instituciones prestadoras de salud hacia el Hospital Pablo Tobón Uribe de Medellín, en el año 2017. 2019;38:114-9.

4. Colombia. Departamento de Cundinamarca. Ministerio de salud y protección social. Decreto 780 del 6 de Mayo de 2016. por medio del cual se expide el Decreto Único Reglamentario del Sector Salud y Protección social. Bogotá. Ministerio de salud y protección social. 2016.

5. Colombia, departamento de Cundinamarca, Ministerio de la protección social. Decreto 4747 del 7 de Diciembre de 2007. Por medio del cual se regulan algunos aspectos de las relaciones entre los prestadores de servicios de salud y de las entidades responsables del pago de los servicios de salud de la población a su cargo, y se dictan otras disposiciones. Bogotá. Ministerio de salud y protección social. 2007.

6. Hirmas J, Poffald L, Jasmen, A, Aguilera X, Delgado I, Vega J. Barreras y facilitadores de acceso a la atención de salud: una revisión sistemática cualitativa. Rev Panam Salud Pública. 2013;33:223-9.

7. Martinez JES. Influencia de los indicadores de gestión en los procesos de referencia y contrareferencia hospitalaria colombiana. 2019;16. 
8. Colombia, departamento de Cundinamarca, ministerio de la protección social. Resolución 3047 del 14 de Agosto de 2008. por medio de la cual se definen los formatos, mecanísmos de envío, procedimientos y términos a ser implementados en las relaciones entre prestadores de servicios de salud y entidades responsables del pago de servicios de salud, definidos en el Decreto 4747 de 2007. Bogotá. Ministerio de salud y protección social. 2008.

9. Lopez F, Muñoz N, Quiroga S, Valero M. Evaluación del tiempo de referencia de II a III nivel de atención del servicio de pediatría en la unidad de servicios de salud de Bosa y el hospital San Rafael de Fusagasugá y las variables que influyen en el período de enero de 2015 a diciembre de 2016. [Trabajo de grado]. [Colombia]: Universidad de Ciencias Aplicadas y Ambientales; 2017.

10. Bravo J, Florez D, Salazar E. Servicios de urgencias en Colombia, el caso de Medellín. [Trabajo de grado]. [Colombia]: Universidad CES; 2008.

11. Colombia. Departamento de Antioquia. Secretaria de salud, Municipio de Medellín. Decreto 0102 del 6 de Febrero de 2009. Por medio del cual se crea el Centro Regulador de Urgencias y Emergencias -CRUE- para el Municipio de Medellín, se implementa el Sistema de Emergencias Médicas-SEM, se modifica el artículo 20 del Decreto Municipal 1240 de 2015 - Consejo Municipal de Gestión del Riesgo de Desastres - CMGRD- y se dictan otras disposiciones. Gaceta oficial, año XXV. No. 4598, 5 de Abril de 2019. Medellín, Secretaria de salud, Alcaldía de Medellín. 2019.

12. Colombia. Departamento de Cundinamarca. Ministerio de salud y protección social. Resolución 926 del 30 de Marzo de 2017, por la cual se reglamenta el desarrollo y operación del Sistema de Emergencias Médicas. Diario Oficial N 50.191 del 30 de Marzo de 2017. Bogotá. Ministerio de salud y protección social. 2017.

13. Colombia, departamento de Cundinamarca, Ministrio de la protección social. Resolución 1220 del 8 de Abril de 2010, Por la cual se establecen las condiciones y requisitos para la organización, operación y funcionamiento de los Centros Reguladores de Urgencias, Emergencias y Desastres, CRUE Diario Oficial N47.679. Bogotá. Ministrior de salud y protección social. 2010. 
14. Bermejo JC, Carabias R, Díaz-Albo Hermida E, Muñoz C, Villacieros M. Derivaciones al Servicio de Urgencias del hospital en una población de ancianos residentes: Estudio retrospectivo sobre sus causas y adecuación. Gerokomos [Internet]. 2010 [citado 20 de septiembre de 2020];21(3). Disponible en: http://scielo.isciii.es/scielo.php?script=sci arttext\&pid=S1134928X2010000300004\&lng=en\&nrm=iso\&tlng=en

15. Herrera J, Conde J, Pinzón L. Evaluación de una Red Integrada de Servicios de Salud en Colombia. Arch Med. 2017;13(3):7.

16. Menéndez JL, Lopez C, Ojalla J, Bouzas E, Cerrada E. Adecuación de las derivaciones desde atención primaria al servicio de urgencias hospitalario en el Área 9 de Madrid. Emergencias: Revista de la Sociedad Española de Medicina de Urgencias y Emergencias. 2005;17(5):215-19.

17. Colombia, departamento de Cundinamarca, ministerio de la protección social. Resolución 8430 del 4 de Octubre de 1993. Por medio de la cual se establecen las normas científicas, técnicas y administrativas para la investigación en salud. Bogotá. Ministerio de salud y protección social. 1993.

18. Schiaffino A, Rodriguez M, Pasarin MI, Regidor E, Borrell C, Fernandez E. ¿Odds ratio o razón de proporciones?: Su utilización en estudios transversales. Gac Sanit. febrero de 2003;17:51-51.

19. Gobernación Departamento del Valle del Cauca. Manual operativo del sistema de referencia y contrarreferencia. [Internet]. 2017. [citado noviembre 2021]. Disponible en:

https://www.google.com/url?sa=t\&rct=j\&q=\&esrc=s\&source=web\&cd=\&ved=2ahUKE wjM aG1lb71AhUiTTABHaN2CawQFnoECAMQAQ\&url=https\%3A\%2F\%2Fwww.valled elcauca.gov.co\%2Fdescargar.php\%3FidFile\%3D558\&usg=AOvVaw1kNUb5QLddpMgG bu9uiDTy

20. Cowling TE, Ramzan F, Ladbrooke T, Millington H, Majeed A, Gnani S. Referral outcomes of attendances at general practitioner led urgent care centres in London, England: retrospective analysis of hospital administrative data. Emerg Med J. marzo de 2016;33(3):200-7. 
21. Cano GS, Gutierrez CAP. Pertinencia de las remisiones y contra remisiones generadas en la red adscrita de servicios de salud de la ciudad de Manizales Caldas durante el año 2013. 2013;79.

22. Bustos B, Villagrán V, Rocha $G$, Riquelme $P$, Muñoz I, Baeza A. Transporte interhospitalario de pacientes pediatricos. Rev Chil Pediatr. 2001;72:430-6.

23. Centro de Proyectos para el desarrollo Cendex. Estudio de disponibilidad y distribución de la oferta de médicos especialistas, en servicios de alta y mediana complejidad en Colombia. [Internet]. 2013. [citado noviembre 2021]. Disponible en https://www.consultorsalud.com/wp-content/uploads/2014/10/disponibilidaddistribucion-md-especialistas-cendex.pdf.

24. Restrepo D, Ortiz L. Aproximaciones a la estimación de la oferta y la demanda de médicos especialistas en Colombia, 2015 - 2030. Observatorio de Talento Humano en Salud. [Internet]. 2017. [citado noviembre 2021]. Disponible en https://www.minsalud.gov.co/sites/rid/Lists/BibliotecaDigital/RIDE/VS/TH/Especialist as-md-oths.pdf.

25. Mueller SK, Shannon E, Dalal A, Schnipper JL, Dykes P. Patient and Physician Experience with Interhospital Transfer: A Qualitative Study. J Patient Saf [Internet]. 2018; Disponible en: http://europepmc.org/abstract/MED/29901654

26. Martín I. Abordaje integral del paciente pluripatológico en atención primaria. Tendencia necesitada de hechos. Aten Primaria [Internet]. Disponible en: https://www.elsevier.es/es-revista-atencion-primaria-27-articulo-abordaje-integraldel-paciente-pluripatologico-S0212656713000292 ER

27. Guidi JL, Golbus JR, Thomas MP, Konerman MC. A Friday Night Transfer. Circ Cardiovasc Qual Outcomes [Internet]. 2019 [citado 20 de septiembre de 2020];12(12). Disponible en: https://www.ahajournals.org/doi/10.1161/CIRCOUTCOMES.119.005856

28. Pronovost PJ. The Weekend Effect in Hospitalized Patients: A Meta-Analysis. J Hosp Med [Internet]. 2017 [citado 20 de septiembre de 2020]. Disponible en: https://www.journalofhospitalmedicine.com/ihospmed/article/145157/hospitalmedicine/weekend-effect-hospitalized-patients-meta-analysis

29. Mueller SK, Fiskio J, Schnipper J. Interhospital Transfer: Transfer Processes and Patient Outcomes. J Hosp Med. agosto de 2019;14(8):486-91. 
30. Handel AE, Patel SV, Skingsley A, Bramley K, Sobieski R, Ramagopalan SV. Weekend admissions as an independent predictor of mortality: an analysis of Scottish hospital admissions. BMJ Open. 2012;2(6):e001789.

31. Cooper A, Davies F, Edwards M, Anderson P, Carson-Stevens A, Cooke MW, et al. The impact of general practitioners working in or alongside emergency departments: a rapid realist review. BMJ Open. 2019;9(4):e024501.

32. van Uden CJT. Does setting up out of hours primary care cooperatives outside a hospital reduce demand for emergency care? Emerg Med J. 2004;21(6):722-3.

33. Pardo L, Zabala C, Gutiérrez S, Pastorini J, Ramirez, Y, Otero S, et al. Sistema de referencia-contrarreferencia en pediatría Análisis de la situación en el Hospital Pediátrico del Centro Hospitalario Pereira Rossell. Rev Méd Urug. 2008;24:69-82.

34. Restrepo JH, Jaén JS, Espinal JJ, Zapata PA. Saturación en los servicios de urgencias: Análisis de cuatro hospitales de Medellín y simulación de estrategias. Gerenc Políticas Salud [Internet]. 2018 [citado 13 de septiembre de 2020]. Disponible en: http://revistas.javeriana.edu.co/index.php/gerepolsal/article/view/23135

35. Marin O, Chavez B. Inconsistencias en la afiliación al sistema de salud colombiano: barrera administrativa para la atención integral de las urgencias médicas. Rev Fac Nac Salud Pública [Internet]. 2014;32(1). Disponible en: https://revistas.udea.edu.co/index.php/fnsp/article/view/13241 